\title{
Lipid domain formation and dynamics in giant unilamellar vesicles explored by fluorescence correlation spectroscopy
}

\author{
Nicoletta Kahya, ${ }^{\text {a,b }}$ Dag Scherfeld, ${ }^{a}$ Kirsten Bacia,,${ }^{a, b}$ and Petra Schwille ${ }^{a, b, *}$ \\ ${ }^{a}$ Experimental Biophysics Group, Max Planck Institute for Biophysical Chemistry, Am Fassberg 11, 37077 Göttingen, Germany \\ b Dresden University of Technology, clo Max Planck Institute of Molecular Cell Biology and Genetics, Pfotenhauerstrasse 108, \\ 01307 Dresden, Germany
}

Received 12 August 2003, and in revised form 4 September 2003

\begin{abstract}
Lipids in eukaryotic cell membranes have been shown to cluster in "rafts" with different lipid/protein compositions and molecular packing. Model membranes such as giant unilamellar vesicles (GUVs) provide a key system to elucidate the physical mechanisms of raft assembly. Despite the large amount of work devoted to the detection and characterization of rafts, one of the most important pieces of information still missing in the picture of the cell membrane is dynamics: how lipids organize and move in rafts and how they modulate membrane fluidity. This missing element is of crucial importance for the trafficking at and from the periphery of the cell regulated by endo- and exocytosis and, in general, for the constant turnover which redistributes membrane components. Here, we review studies of combined confocal fluorescence microscopy and fluorescence correlation spectroscopy on lipid dynamics and organization in rafts assembled in GUVs prepared from various lipid mixtures which are relevant to the problem of raft formation.
\end{abstract}

(c) 2003 Elsevier Inc. All rights reserved.

Keywords: Fluorescence correlation spectroscopy; Confocal fluorescence microscopy; GUVs; Lipid rafts; Cholesterol; Phosphatidylcholine; Sphingomyelin

A great number of cellular functions are associated with biological membranes. Although each cellular membrane has a unique set of proteins, which carry out mass transport or signal transduction, the basic building blocks are lipids. These amphipathic molecules provide the mechanical stability and the low permeability to ions or big molecules. Importantly, they are also able to actively manipulate the functionality of proteins embedded in the membrane matrix, directly via specific lipidprotein interactions or indirectly via changes in collective properties such as flexibility, surface tension, and lateral pressure (Sprong et al., 2001). It is thus straightforward that many types of lipids are needed to tailor the physical properties of a given membrane bilayer. One important point that should be addressed in membrane studies is the highly dynamic traffic to and within cellular membranes (Edidin, 2003) and lipid

\footnotetext{
${ }^{*}$ Corresponding author. Fax: +49-551-201-1409.

E-mail address: schwille@mpi-cbg.de (P. Schwille).
}

lateral motions, which ensure enzymatic turnovers, recycling, and aggregation processes. Extensive research, following the raft hypothesis for the membrane structure formulated 15 years ago by Simons and colleagues (Simons and van Meer, 1988), has focused much interest on the lipid spatial organization in the membrane matrix and the functional mechanisms of lipid clustering. More recently, along with a number of techniques employed to address questions on rafts (Brown and Rose, 1992; Fridriksson, 1999; Heerklotz, 2002), important contributions have also come from optical microscopy (Kenworthy et al., 2000; Schütz et al., 2000). Direct visualization of raft-like domains in model bilayer membranes has provided a tangible proof for the coexistence of liquid-ordered and liquid-disordered phases (Bagatolli et al., 2000; Dietrich et al., 2001a,b). However, in these studies the domain morphology is characterized only from a static point of view. On the contrary, rafts are by no means static structures. If their main function consists of forming platforms to 
concentrate certain proteins and exclude others, then a detailed characterization of lipid and protein dynamics in the different phases is essential to understand mobility-dependent protein organization (Simons and Toomre, 2000). Single-particle tracking (SPT) has been applied to follow raft-associated proteins in vivo (Schütz et al., 2000) and lipid mobility in cell membranes and in vitro (Fujiwara et al., 2002). Additional contributions have come from fluorescence recovery after photobleaching (FRAP) (Dietrich et al., 2001a,b) and fluorescence resonance energy transfer (FRET) (Kenworthy et al., 2000). However, dynamics at the molecular level in raft-exhibiting membranes is still poorly understood.

Fluorescence correlation spectroscopy (FCS) exploits the temporal dimension of fluorescence fluctuations induced by changes in molecular fluorescence properties in a small open volume in the sample. A wide range of dynamic parameters can be explored, such as translational and rotational diffusion coefficients, association/ dissociation constants, and lifetimes of excited states (e.g., triplets). Unlike FRAP, FCS works at the singlemolecule level (sensitivity down to nanomolar concentrations), which makes the technique less invasive and more reliable because of the minimal disturbance of the system under investigation. Furthermore, FCS data with high statistical accuracy are achieved in much shorter times than SPT studies. FCS has been successfully applied to diffusion studies in cells (Bacia et al., 2002; Schwille et al., 1999) and artificial membranes (Fahey et al., 1977; Korlach et al., 1999). In particular, when applied to well-defined bilayer systems such as giant unilamellar vesicles (GUVs), FCS has been proven to characterize the molecular mobility in distinct lipid phases, providing information about the physico-chemical properties as well as lipid composition within specific domains (Kahya et al., 2003; Scherfeld et al., 2003).

In the present review, we first describe the properties of GUVs and illustrate the advantages of using GUVs for confocal microscopy. After an introduction to the principle and experimental setup of FCS, we describe how to measure lateral diffusion coefficients of lipids in GUV membranes and then focus on lipid dynamic properties in GUVs made from binary lipid mixtures (phospholipid/cholesterol). When domains assemble in GUVs, FCS allows for investigating how lipids organize and diffuse within domains and the role played by cholesterol in the regulation of lipid dynamics in various ternary lipid mixtures, which are relevant to the raft formation, as discussed in the last two sections.

\section{Model membranes for light microscopy: giant unilamellar vesicles}

Biomolecular membrane research has a long tradition of development and application of artificial membranes as a model for complex biological membranes. To date, several model membranes have been made available for different purposes (Sackmann, 1996). In principle, good model membranes should consist of a closed, spherical single bilayer whose curvature matches that of (intra)cellular membranes. The bilayer structure should be regular and stable and have the appropriate properties of conductance, dielectric constant, and fluidity. It would also be desirable to have integral or surface-associated peripheral membrane proteins inserted in the bilayer, such that the correct folding, activity, aggregation state, and lateral dynamics are maintained. Depending on the purposes and technical limitations, all the model membranes described thus far lack one or more of these properties. Among artificial membrane systems available for (single-molecule) optical microscopy (e.g., monolayers, black lipid membranes (BLMs), supported planar bilayers), GUVs represent an excellent choice.

Monolayers (Lipp et al., 1996) are easy to prepare and provide a regular, fluid, and stable structure. However, their ability to mimic biomembranes can be questioned, as they lack the second leaflet to form a bilayer. On the other hand, BLMs (Jain, 1972) often do not present a regular structure and unilamellarity, as there are many technical limitations which prevent formation of an ideal BLM. As an alternative to BLMs, planar-supported bilayers were developed in the early 1980s (von Tscharner and McConnell, 1981) and consist of membranes assembled at the interface between a solid substrate and an aqueous phase. However, lipid-substrate interactions and/or the rather low equilibrium thickness of the intermediate water layer are sources of potential artifacts, in particular when integral proteins are inserted in the bilayer. Parts of the soluble residues might (strongly) interact with the solid substrate, leading to partial or complete loss of dynamics and functionality. Actually, many biological reactions in membranes depend on the lateral mobility and spatial distribution of the proteins. Therefore, to bear potential biological relevance, a model membrane should provide an environment which ensures a close-to-native dynamics and mobility of the protein. Potentially, the artifacts present in supported membranes are totally absent in GUVs, a rapidly emerging bilayer model in the membrane research field (Menger and Keiper, 1998). GUVs are spherical closed bilayers, freely standing in aqueous solution. They are suitable for single-molecule optical microscopy and exhibit a cell-like curvature, as their size ranges from 10 upto $100 \mu \mathrm{m}$ in diameter.

In our hands, the most appropriate and convenient procedure for preparing GUVs is a modification of the electroformation method (Angelova and Dimitrov, 1986; Angelova et al., 1992), which produces truly unilamellar vesicles with a high yield (Kahya et al., 2003; Scherfeld et al., 2003). Briefly, a thin lipid layer is dried 
on a conductive glass coverslip (indium tin oxide, ITO) coated. The sample chamber, composed of two ITOcoated glass plates sealed by a Teflon spacer, is closed and filled with the buffer medium. An AC voltage is then applied between the glass plates, inducing a lipid swelling and, in the end, creating unilamellar giant liposomes. GUVs can be prepared with a large variety of phospholipids and mixtures thereof, an important limitation being only that high ionic strengths of the buffer decrease the efficiency of vesicle formation.

As a result of their advantages compared to other model membranes, GUVs are becoming increasingly important in several branches of membrane research. The structure and variety of their shapes reflect the physical nature of lipid molecules and their environment, allowing for a description of several static (e.g., curvature energy, surface tension, geometrical constraints for surface area and volume, which result in a preferential vesicle shape) and dynamic properties (e.g., thermal undulations) (Seifert, 1997). Changes in morphology and composition can be readily observed upon addition of surfactants (Needham et al., 1997) and polysaccharides (Ueda et al., 1998). Biochemical interactions can be dissected for membrane-interacting enzymes (Wick et al., 1996; Holopainen et al., 2000), either on the single vesicle or at the single-molecule level. Giant vesicle micromanipulation allows for investigation of various time-dependent (bio)chemical, mechanical, and thermal perturbations. Finally, GUVs can be regarded as stripped-down biomembranes and thus provide an excellent model to study cytomimetic phenomena (e.g., fusion, fission, budding, exo- and endocytosis) under controllable conditions.

\section{Fluorescence correlation spectroscopy: principles and experimental setup}

Fluorescence correlation spectroscopy (Eigen and Rigler, 1994; Magde et al., 1972; Schwille, 2001) relies on the possibility of detecting single molecules and investigating their dynamic properties, which are observed over a wide temporal acquisition window (from submicroseconds up to seconds). The fluorescence intensity fluctuations occurring at equilibrium carry information about the average number of molecules and characteristic time scales of any dynamic events that determine the rise and decay of the fluorescence signal. Possible applications include the determination of local concentrations, molecular weights, translational/rotational diffusion coefficients, chemical rate constants, association/dissociation constants, inter/intramolecular interactions, and photodynamics (e.g., triplet state relaxation).

The classical method to study mobility of particles in two and three dimensions is FRAP (Axelrod et al., 1976), which requires heavy-loaded labeling and high laser powers, whereas FCS offers a much less invasive alter- native method with limited photodamage and toxicity for the sample. Compared to SPT (Saxton and Jacobson, 1997), which also provides information on dynamic parameters such as diffusion and aggregation constants, FCS gives a good statistical accuracy in shorter times (Koppel, 1974). An extension of FCS, dual-color crosscorrelation spectroscopy (Rarbach et al., 2001; Schwille et al., 1997), accesses coincident molecular events of different species such as codiffusion of binding partners, offering a tool of great potential for studying molecular interactions with minimal disturbance of the system. For the same purpose, other more traditional optical techniques have been employed to detect protein association, such as colocalization by confocal imaging and FRET. While these techniques exploit the dimension of space, correlation spectroscopy involves the dimension of time, probing the concomitant movement of differently labeled particles through the focus. This avoids artifacts that could arise from a random colocalization of the particles without mutual interaction and does not require the close proximity of the labels, like FRET.

The normalized autocorrelation function $G(\tau)$ of the temporal fluctuations of the fluorescence $F(t)$ is given by (Schwille, 2001)

$$
G(\tau)=\frac{\langle\delta F(t+\tau) \delta F(t)\rangle}{\langle F(t)\rangle^{2}}=\frac{\langle\delta F(\tau) \delta F(0)\rangle}{\langle F\rangle^{2}} .
$$

In the simplest case, in which only one optical species is present in the focal volume, the fluorescence intensity can be expressed as

$F(t)=\kappa Q \int W(r) C(r, t) \mathrm{d} \Omega$,

where $C(r, t)$ is the number density at position $r$ and time $t, Q$ is the product of the absorption coefficient and fluorescence quantum efficiency of the fluorescent molecules, $W(r)$ defines the detection area, and $\kappa$ is the overall fluorescence detection efficiency.

If we consider that the focal volume and its illumination is fixed and stable, the temporal fluorescence fluctuations can be caused only by variations in concentration at position $r$ and time $t$ from the average value $\langle C\rangle$,

$\delta C(r, t)=C(r, t)-\langle C\rangle$,

and the fluorescence fluctuation at time $t$ can be rewritten:

$\delta F(t)=\kappa Q \int \delta C(r, t) W(r) \mathrm{d} \Omega$.

Generalizing to a system of multiple noninteracting particles, the normalized autocorrelation function of the fluorescence fluctuations is thus equal to

$$
G(\tau)=\frac{\sum_{i=1}^{R} \sum_{j=1}^{R} Q_{i} Q_{j} \iint W(r) W\left(r^{\prime}\right) f_{i j}\left(r, r^{\prime}, \tau\right) \mathrm{d} \Omega \mathrm{d} \Omega^{\prime}}{\left[\sum_{i=1}^{R} Q_{i}\left\langle C_{i}\right\rangle \int W(r) \mathrm{d} \Omega\right]^{2}},
$$


where the term

$$
\begin{aligned}
f_{i j}\left(r, r^{\prime}, \tau\right) & =\left\langle\delta C_{i}(r, t+\tau) \delta C_{j}\left(r^{\prime}, t\right)\right\rangle \\
& =\left\langle\delta C_{i}(r, \tau) \delta C_{j}\left(r^{\prime}, 0\right)\right\rangle,
\end{aligned}
$$

represents the correlation function of the concentration fluctuation in the $i$ th chemical species at position $r$ with a concentration fluctuation in the $j$ th chemical species at position $r^{\prime}$ and a time $\tau$ earlier. The value $G(0)$ depends on the average particle number and relative quantum yield of the fluorescence species and on the shape of the detection volume.

When FCS is performed on particles of one species freely diffusing in 2D (on a membrane surface) with diffusion coefficient $D_{i}$, the term $f_{i j}(r, r, \tau)$ reduces to

$f_{i}\left(r, r^{\prime}, \tau\right)=\left(4 \pi D_{i} \tau\right) \exp \left[-\left|r-r^{\prime}\right|^{2} /\left(4 D_{i} \tau\right)\right]$.

Assuming the focal volume as a Gaussian function with $1 / e^{2}$ dimension $r_{0}$, the autocorrelation function can be written as (Magde et al., 1972)

$G(\tau)=\frac{\left(\sum_{i}\left\langle C_{i}\right\rangle\left(\frac{1}{1+\tau / \tau_{d, i}}\right)\right)}{A_{\mathrm{eff}}\left(\sum_{i}\left\langle C_{i}\right\rangle\right)^{2}}$,

where $\left\langle C_{i}\right\rangle$ is the two-dimensional time average concentration of the species $i$ in the detection area $A_{\text {eff }}=\pi r_{0}^{2}$ $\left(\sim 0.1 \mu \mathrm{m}^{2}\right)$, and $\tau_{d, i}$ is the average residence time of the species $i$. The diffusion coefficient $D_{i}$ for the species $i$ is inversely proportional to $\tau_{d, i}$ with $\tau_{d, i}=r_{0}^{2} / 4 D_{i}$.

A typical FCS scheme is shown in Fig. 1. A tight spatial confinement of the sample is needed for a resolution of small molecular ensembles. Therefore, the core of a microscope setup for FCS is a high numerical aperture objective, through which a diffraction-limited illumination is achieved. The laser beam passes through a spatial filter and is reflected by a dichroic mirror before being focused by the objective. Fluorescence is collected back through the same optical path and is transmitted by the dichroic mirror. A lens focuses the signal into a pinhole in front of the detector (a photomultiplier or an avalanche photodiode) to limit out-of-focus detection. In cases in which two optical species (with different emission properties) are followed at the same time, a

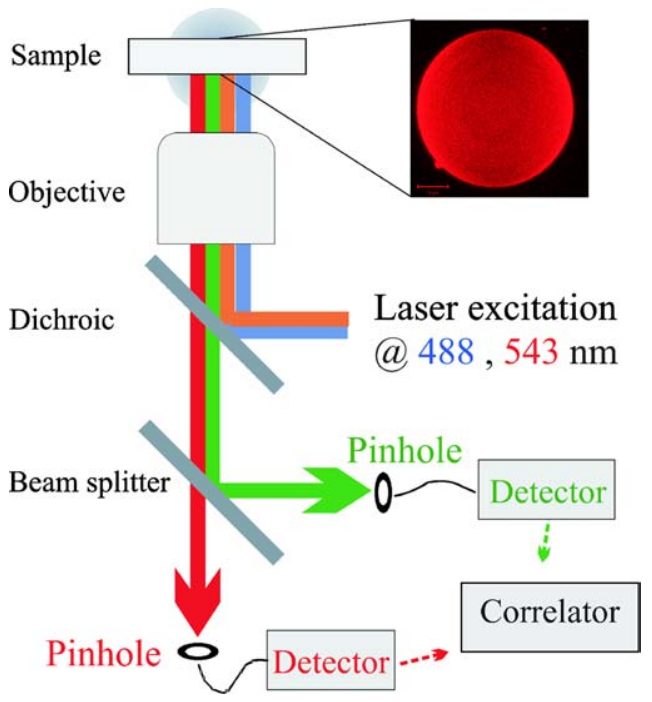

Fig. 1. Optical setup of confocal fluorescence microscopy for FCS and imaging. The laser excitation light is focused onto the sample via a high-numerical aperture objective (parallel geometry), after being reflected by the dichroic mirror. The fluorescence emission is recollected by the same objective and transmitted by the dichroic. Fluorescence can be spectrally separated by a beam splitter (i.e., for dual-color confocal fluorescence imaging) before being projected onto the detector(s). The confocal geometry (ensured by pinholes in front of the detectors) reduces the background and increases the signal-to-noise ratio.

second dichroic mirror splits the fluorescence signal, allowing for the simultaneous collection of the distinct portions of the fluorescence spectrum with two detectors. In some applications, such as the study of lipid and protein dynamics in membranes, the position of the focus with respect to the source of signal is critical for the recording of correlation curves without artifacts and the interpretation of the data (see the following section). Therefore, it is always recommended to combine a FCS setup with a laser scanning module, as provided in commercial FCS solutions (e.g., Zeiss ConfoCor II used for the below-reported applications). In general, a compromise in the concentration range can be found that supports both applications, i.e., an acceptable contrast quality in confocal imaging and good amplitude of correlation function in FCS.

Fig. 3. Binary mixtures of DOPC/cholesterol and DLPC/cholesterol exhibit a continuous change in diffusion coefficient as a function of cholesterol concentration. Average diffusion coefficients, as determined from the fitting of the autocorrelation curves shown in insets A (DOPC/cholesterol, in red for $0 \%$, magenta for $20 \%$, light gray for $33 \%$, gray for $50 \%$, and dark gray for $67 \%$ cholesterol) and B (DLPC/cholesterol, in red for $0 \%$, magenta for $20 \%$, light gray for $33 \%$, gray for $50 \%$, and dark gray for $67 \%$ cholesterol), are reported as a function of cholesterol concentration (DOPC/ cholesterol in red, DLPC/cholesterol in black). Bars represent the standard deviation from the average values.

Fig. 5. (A) 3D projection reconstructed from confocal slices $(\sim 0.4 \mu \mathrm{m}$ thick) of GUVs composed of SM/DOPC/cholesterol $1 / 1 / 1$. DiI-C 18 (red) strongly favored the DOPC-enriched, fluid-disordered phase, whereas Alexa Fluor-labeled cholera toxin (AF-CTB, green) bound to GM1 in GUV areas, from which DiI-C 18 was excluded. (B) 3D projection reconstructed from confocal slices ( $\sim 0.4 \mu \mathrm{m}$ thick) of GUVs composed of SM/DOPC 0.5/ 0.5 and cholesterol $50 \%$. Fluorescence from DiI-C 18 (see inset, red) and GM1-bound AF-CTB (see inset, green) is homogeneously distributed on the GUV surface. (C and D) 3D projection reconstructed from confocal slices ( $\sim 0.4 \mu \mathrm{m}$ thick) of GUVs composed of DOPC/DPPC 0.5/0.5 and cholesterol 20\% (C) and DOPC/DSPC 0.5/0.5 and cholesterol 20\% (D). 


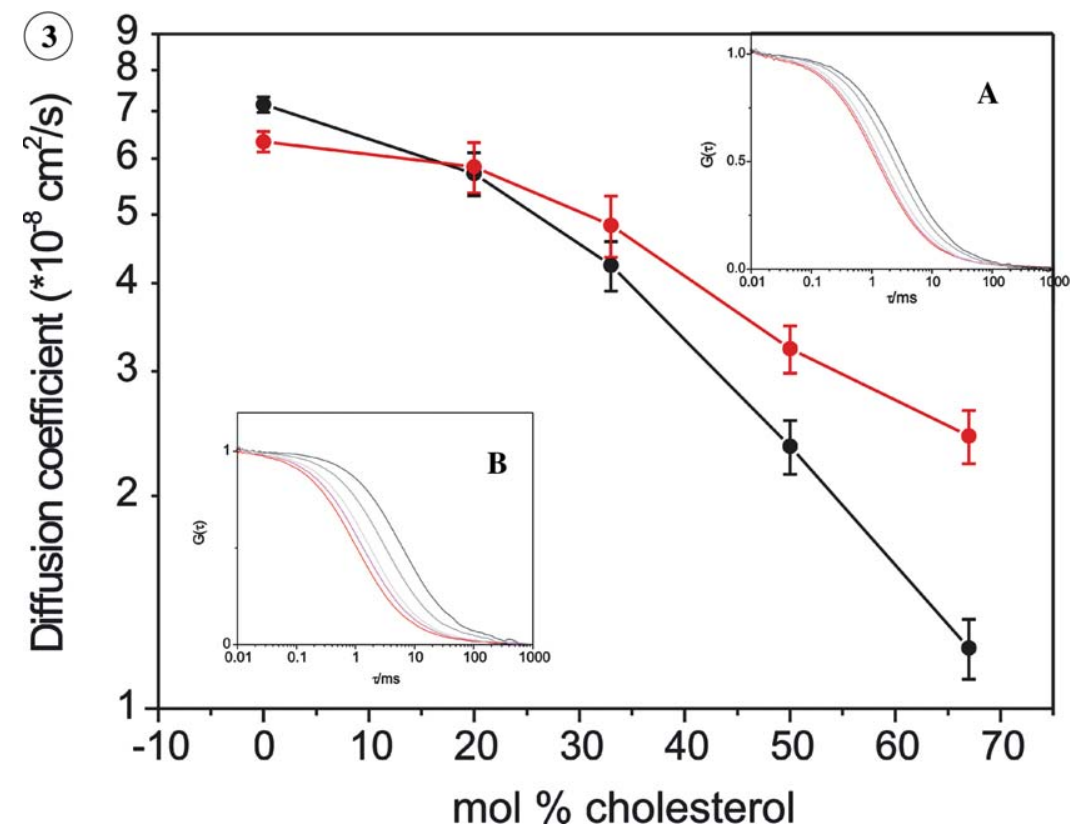

(5)
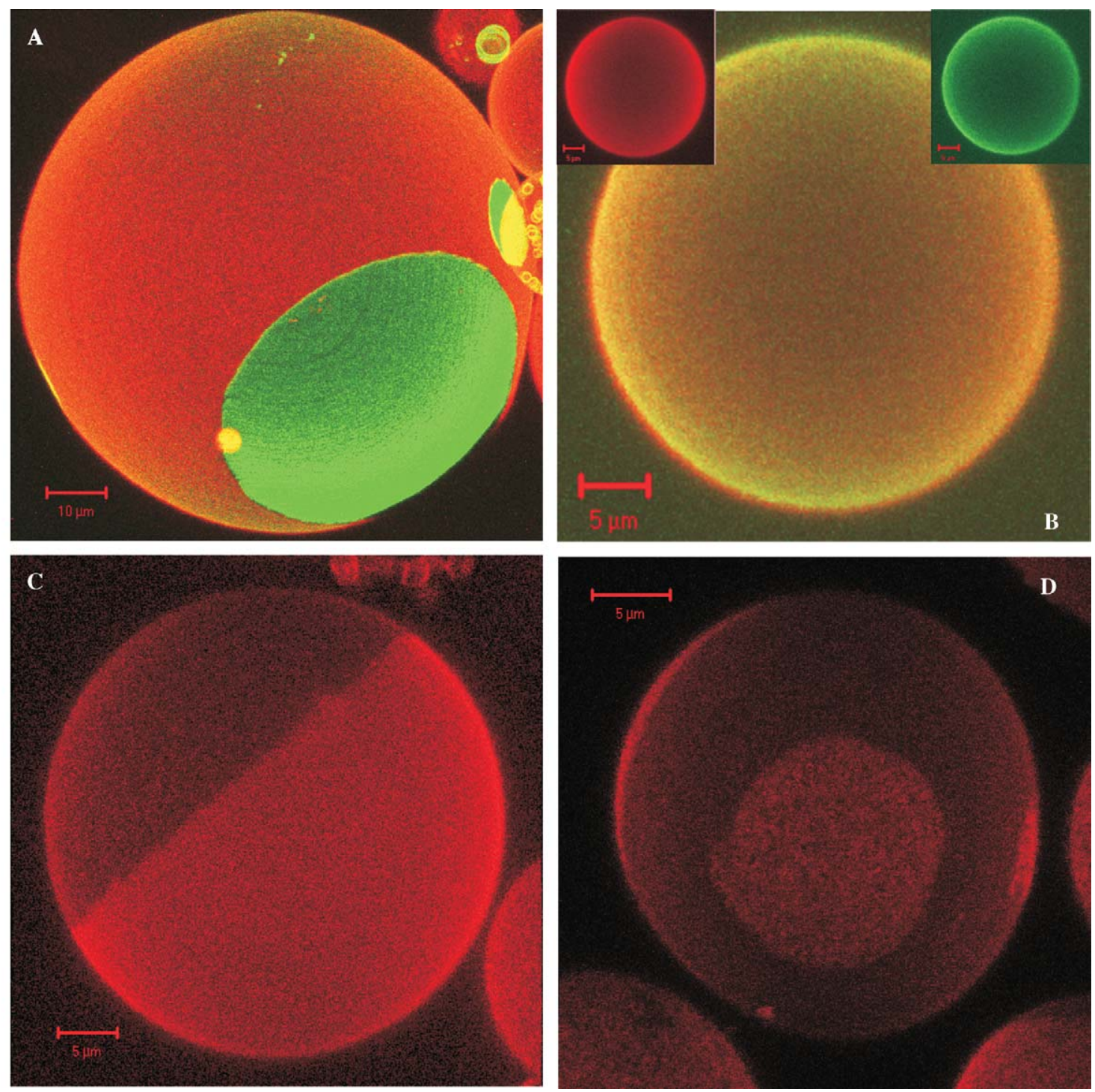

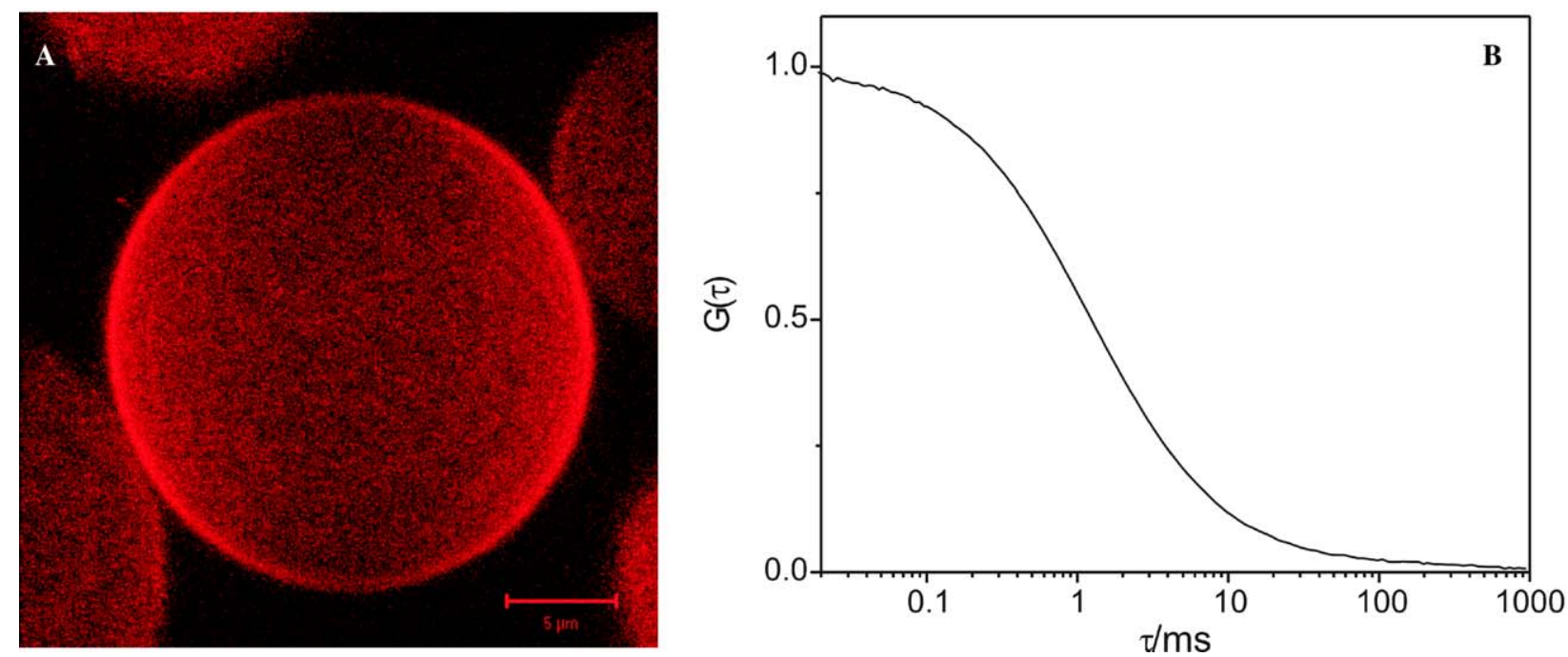

Fig. 2. (A) 3D projection of a GUV (DOPC with $0.1 \mathrm{~mol} \%$ DiI- $\mathrm{C}_{18}$ ), obtained from confocal slices ( $\sim 0.4 \mu \mathrm{m}$ thickness), which shows homogeneous fluorescence corresponding to a single fluid phase. (B) Autocorrelation curve recorded from fluorescence fluctuations of $\mathrm{DiI}-\mathrm{C}_{18}(0.001 \mathrm{~mol} \%)$ in DOPC GUVs, which can nicely be fitted by a single diffusing species.

\section{Lipid translational diffusion in giant unilamellar vesicles}

FCS has been successfully applied to study diffusion of lipids in membranes (Fahey et al., 1977; Korlach et al., 1999; Schwille et al., 1999, 2001). In Fig. 2A, a 3D projection obtained by confocal images at different $z$ positions of a GUV composed of pure DOPC at room temperature is shown. By focusing the laser light on the top (bottom) side of GUVs, FCS curves are recorded, which give information on the lateral diffusion of lipophilic probes incorporated in the membrane and moving in and out of focus. Fig. 2B shows the FCS curve obtained from fluorescence temporal fluctuations from DiI- $C_{18}$ in DOPC GUVs $\left(D=6.3 \pm 0.2 \times 10^{-8} \mathrm{~cm}^{2} / \mathrm{s}\right)$. Lateral diffusion can be modeled as a two-dimensional Brownian motion.

Owing to the ability of FCS to observe particles in diluted solutions, diffusion of particles inserted in GUVs can be studied at the single-molecule level. This is a great advantage in studies on domain formation in membranes, as (i) lipid analogs can be introduced in minimal amounts and (ii) at the single-molecule level, clustering of the dye may be readily detected. Furthermore, FCS has been shown to be sensitive to deviations from single-phase behavior, e.g., caused by heterogeneities in the sample (Korlach et al., 1999). For the sake of accuracy in the data analysis, the focal spot is positioned at the top/bottom of a GUV, which is large enough to be considered approximately planar $\left(\sim 0.1 \mu \mathrm{m}^{2}\right)$ in the focus. Precautions need to be taken to rule out potential severe artifacts arising from the inaccurate positioning of the detection volume with respect to the bilayer. First of all, the optimal $x, y, z$ position of the top/bottom side of GUVs is chosen by high-resolution confocal scanning microscopy. Routine tests are usually performed to control the lipid diffusion times as a function of the bilayer position with respect to the focus, as well as a function of the count rate per particle (see for ex-

Fig. 6. (A) Average diffusion coefficients, as determined from fitting the autocorrelation curves as a function of cholesterol concentration (Kahya et al., 2003). Values for the DOPC-enriched phase are shown in red, those for the SM-enriched phase and for mixtures that do not give rise to phase separation (within the optical resolution) are indicated in black. The solid red line, which corresponds to the value of lipid diffusion coefficient in GUVs of DOPC, is shown as a reference. (B) Scheme of the ternary phase diagram for the SM/DOPC/cholesterol system at $25^{\circ} \mathrm{C}$, constructed on the basis of confocal imaging and FCS data (Kahya et al., 2003). The highlighted region indicates the areas at which phase separation occurs. Circles refer to the compositions analzed for the SM/DOPC/cholesterol system (filled circles indicate no phase separation visible in the confocal microscope; P. coexistence of liquid-disordered and liquid-ordered phases, hence, raft-like domains; 9 , pure gel phase; $\Theta$, coexistence of gel phase and liquiddisordered phase; $Q$ coexistence of gel phase and liquid-ordered phase). Numbers next to the circles give the average lipid diffusion coefficients $\left(\times 10^{-8} \mathrm{~cm}^{2} / \mathrm{s}\right)$. Insets report $3 \mathrm{D}$ projection reconstructed from confocal slices $(\sim 0.4 \mu \mathrm{m}$ thick) of GUVs composed of DOPC (bottom left), DOPC/ SM 0.5/0.5 and 50\% cholesterol (upper left), DOPC/SM/cholesterol 1/1/1 (upper right), DOPC/SM 0.5/0.5 and 20\% cholesterol (middle right), and $\mathrm{SM}$ (bottom right). Red, fluorescence from DiI-C 18 ; green, fluorescence from a GM1-bound fluorescent conjugate of cholera toxin B subunit. Bars, $10 \mu \mathrm{m}$. See Kahya et al. (2003).

Fig. 7. Average diffusion coefficients, as determined from fitting the autocorrelation curves, as a function of cholesterol concentration, for (A) DOPC/ DPPC 0.5/0.5 and cholesterol and (B) DOPC/DSPC 0.5/0.5 and cholesterol mixtures (Scherfeld et al., 2003). Red symbols indicate the phase favored by DiI- $\mathrm{C}_{18}$, black symbols indicate domains from which the probe is excluded, and gray symbols indicate single-phase GUVs. 
(6)
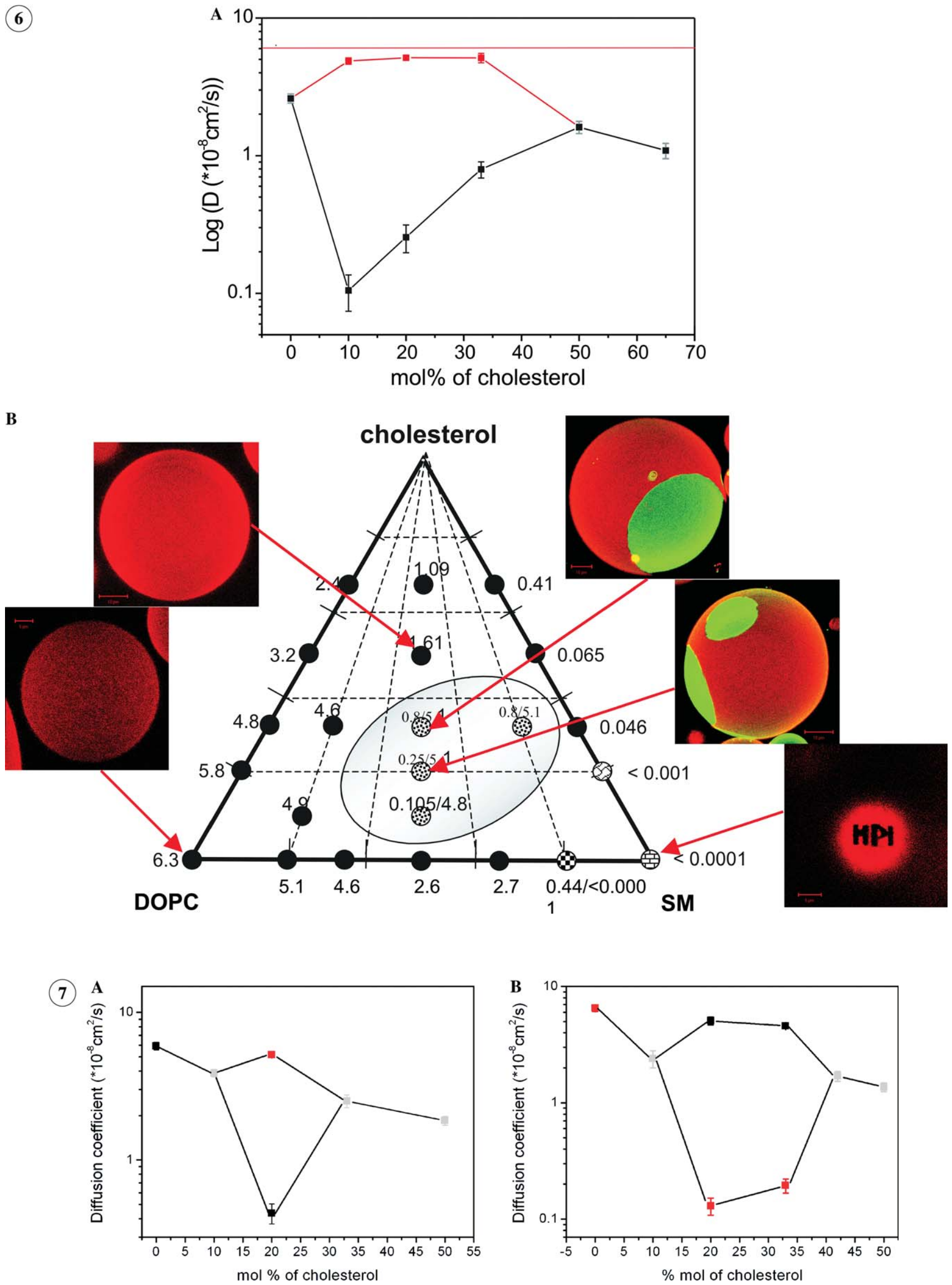
ample Milon et al., 2003). During the acquisition of FCS data, the fluorescence count rate is monitored online, ensuring that no fluorescence fluctuations originate from movements of the whole membrane. Finally, we prepare fresh vesicles for every FCS experiment. When freshly prepared, GUVs stay nicely in tension for approximately $2-3 \mathrm{~h}$, thereby providing a planar tense bilayer in the focal spot. The consistency of the diffusion times and the single-phase behavior of the FCS curves sufficiently prove that no artifacts compromise the data. Sometimes, thermal undulations of the bilayer in focus induce an additional component in the FCS curve, characterized by long (with respect to the real lipid diffusion time) and variable diffusion times. Data affected by such artifacts should be rejected.

In the following sections, a few applications of FCS combined with confocal scanning microscopy are presented, to study lipid-lipid and lipid-sterol interactions in binary mixtures and raft assembly and effects of cholesterol on lipid dynamic organization in ternary mixtures.

\section{Lipid dynamics in binary lipid/sterol mixtures}

More than 30 years of research, using a variety of thermodynamic and spectroscopic techniques, have helped to clarify the nature of lipid/cholesterol interactions but left many gaps in the understanding of the distinctive features of such very complex interactions (for a review see McConnell and Vrljic, 2003; Silvius et al., 1996; Silvius, 2003, see also Huang et al., 1999; Feigenson and Buboltz, 2001). When cholesterol is added to phospho- and/or sphingolipids, it adopts complex patterns of lateral organization, which greatly affect the molecular mobility in the plane of the bilayer (McConnell and Radhakrishnan, 2003; McConnell and Vrljic, 2003). As postulated by Ipsen et al. (1987), cholesterol and phospholipids form a liquid-ordered $\left(l_{\circ}\right)$ phase characterized by a high level of molecular order in the lipid packing and a high degree of diffusion mobility (Sankaram and Thompson, 1991; Vist and Davis, 1990). The $1_{o}$ phase could coexist with the liquid-disordered phase (or liquid-crystalline, $1_{d}$ ), characterized by a high degree of disorder and very high lipid mobility, and with the gel phase, in which lipid molecules are virtually immobile. The concept of the $1_{0}$ phase has provided a possible tool to describe the nature of lipid rafts in biological membranes (Brown and London, 1998).

FCS has been used to probe how molecular interactions in membranes affect lipid dynamic properties (A1meida et al., 1993; Kahya et al., 2003; Korlach et al., 1999). In our laboratory, the effect of cholesterol on the dynamic properties of lipids with low phase-transition temperatures $\left(T_{\mathrm{m}}\right)$, such as DOPC $\left(T_{\mathrm{m}}=-20^{\circ} \mathrm{C}\right)$ and DLPC $\left(T_{\mathrm{m}}=8^{\circ} \mathrm{C}\right)$ has been recently investigated (Ka-

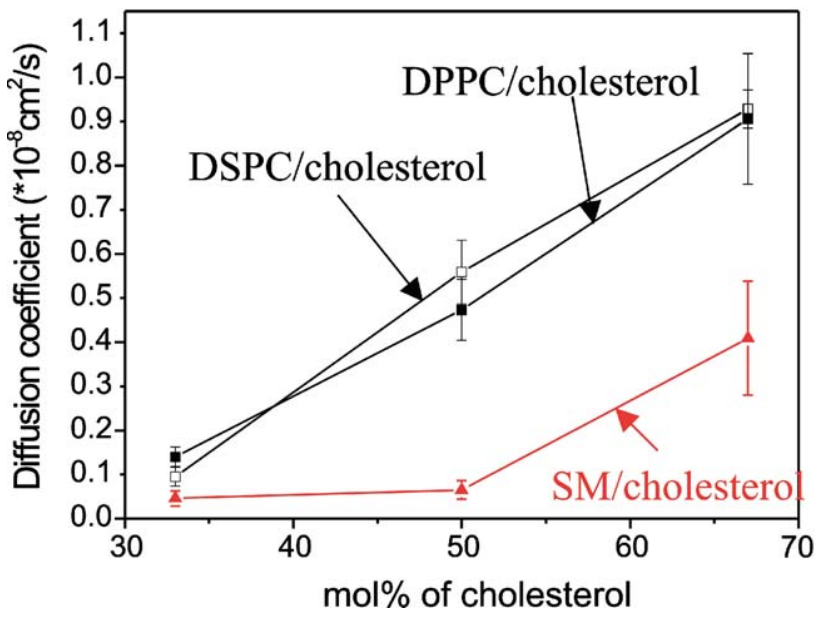

Fig. 4. Binary mixtures of SM/cholesterol, DPPC/cholesterol, and DSPC/cholesterol exhibit an increase in diffusion coefficient as a function of cholesterol concentration. Average diffusion coefficients, as determined from the fitting of the autocorrelation curves (Kahya et al., 2003), are reported as a function of cholesterol concentration. Bars represent the standard deviation from the average values. In the absence of cholesterol, SM (DPPC, DSPC) was in the gel phase and the lipid translational mobility was very limited (Kahya et al., 2003). Also, the lipid mobility at $\leqslant 33 \mathrm{~mol} \%$ cholesterol was too low to be measured by FCS.

hya et al., 2003; unpublished results). When GUVs composed of DOPC/cholesterol and DLPC/cholesterol in various ratios are imaged confocally, by using the fluorescent probe $\mathrm{DiI}-\mathrm{C}_{18}$, no phase separation is observed at room temperature. This implies a rather homogeneous bilayer structure, and heterogeneities, if present, are smaller than the optical resolution $(\sim 300 \mathrm{~nm})$. Accordingly, molecular lateral diffusion appears homogeneous, as illustrated by single-phase FCS curves fitted to a 2D Brownian diffusion model (Fig. 3). The gradual decrease in lipid mobility observed for both DOPC and DLPC as a function of sterol amount is consistent with the observations reported in literature about the "condensing" effects of cholesterol on low $T_{\mathrm{m}}$ phospholipids (Demel and de Kruijff, 1976). The absence of steep changes in the diffusion coefficient suggests that the transition from a region of composition with high lipid mobility $\left(l_{d}\right.$ phase $)$ to one with lower mobility $\left(l_{o}\right.$ phase) is continuous. Furthermore, the decrease in lipid mobility is steeper for DLPC than for DOPC, implying that cholesterol engages stronger interactions with the saturated phospholipid that with the unsaturated one. These conclusions are consistent with previous experimental observations (Brzustowicz et al., 2002; OhvoRekilä et al., 2002). In an earlier FCS study (Korlach et al., 1999), dynamics in giant vesicles composed of DLPC/cholesterol mixtures were investigated, yielding a consistent trend of $\mathrm{D}$ as a function of sterol amount. Slightly different values for the diffusion coefficients were found, most likely due to a different membrane preparation procedure (yielding, e.g., multilamellar vesicles). 
We also investigated mixtures of cholesterol and high $T_{\mathrm{m}}$ lipids, i.e., SM (Kahya et al., 2003), DPPC, and DSPC (Scherfeld et al., 2003). Confocal fluorescence microscopy showed that for all of the lipid compositions analyzed, fluorescence from DiI- $\mathrm{C}_{18}$ is homogeneously distributed on the GUV surface. Consistent with optical imaging, FCS experiments revealed a homogeneous Brownian-like lipid diffusion behavior (see Fig. 4). In contrast to low $T_{\mathrm{m}}$ lipids, cholesterol increases lipid mobility in SM, DPPC, and DSPC membranes, thereby exerting a "fluidizing" effect on the membrane. Both the absolute values and the trend of the lipid diffusion coefficients for the saturated phospholipids DPPC and DSPC are, within the experimental error, coincident, implying that a change in the PC chain length by two carbon atoms does not affect the interaction energy with cholesterol (Scherfeld et al., 2003). Most importantly, the observed changes in diffusion coefficients as a function of sterol content in PC/cholesterol membranes are only qualitatively and not quantitatively similar to those in SM/cholesterol bilayers (see Fig. 4; Kahya et al., 2003). In the case of SM, the lipid mobility is lower than that of PC with comparable saturated chain length, i.e., DPPC and DSPC (see Fig. 4). Overall, the FCS data clearly indicate that cholesterol interacts more strongly with SM than with saturated phospholipids with matched chain length. This interpretation supports a molecular model of lipid/sterol interactions, according to which a strong network of hydrogen bonds created by $\mathrm{SM}$ but not by $\mathrm{PC}$ is responsible for the higher stiffness of SM membranes with respect to PC bilayers (OhvoRekilä et al., 2002). The lipid-lipid interactions are disrupted when cholesterol inserts into the bilayer and, as a consequence, the mobility of the headgroup is enhanced (Yeagle et al., 1977). As the sphingolipid/ cholesterol interaction is stronger than the phospholipid/ cholesterol interaction, cholesterol intercalates more tightly in SM bilayers than in phospholipid bilayers, producing a smaller "fluidizing" effect in the former system and, as a consequence, a lower lipid mobility.

Overall, we have shown that FCS combined with confocal imaging provides a valuable tool to probe the strength of lipid-sterol interactions and gives insight into the energetics underlying the lipid spatial distribution in membrane bilayers.

\section{Raft assembly visualized in giant unilamellar vesicles}

Significant evidence has been presented on the existence of lipid rafts in membranes enriched in sphingolipids and cholesterol in the liquid-ordered phase (Simons and Ikonen, 1997; Simons and van Meer, 1988). Although conventional techniques to "purify" rafts, e.g., detergent extraction of cell membranes, were first employed to study raft lipid composition, they might be affected by artifacts
(Brown, 2001; Brown and London, 2000). Optical microscopy allows for a straightforward visualization of lipid domains in a noninvasive way (Bagatolli and Gratton, 1999, 2000). By detecting fluorescence from lipid probes expected to partition preferentially either into rafts or into nonraft phase, raft-like domains could be visualized in monolayers and supported bilayers (Dietrich et al., 2001a,b; Yuan et al., 2002). Importantly, raft assembly has been proven to occur also in free-standing, close-tonative model membranes, i.e., GUVs (Dietrich et al., 2001a,b; Keller et al., 2002), ruling out artifacts that could possibly arise in other model membranes. A systematic investigation of the morphology of raft-like domains as a function of cholesterol concentration has been recently attempted in our laboratory (Kahya et al., 2003). We have employed confocal fluorescence microscopy to visualize domain formation in GUVs and investigated the changes in lipid spatial organization upon systematic variation of sterol content in ternary SM/DOPC/cholesterol systems at room temperature. In these experiments, a fluorescent marker, DiI- $\mathrm{C}_{18}$, was used, which has been often used in mixtures of saturated phospholipids and has been shown to partition preferentially with saturated, long-tailed phospholipids, e.g., in DPPC phases over coexisting fluid phases by a factor of $\sim 3$ (Spink et al., 1990). We showed that $\mathrm{DiI}-\mathrm{C}_{18}$ is excluded from the sphingolipid-rich phase and rather favors the DOPC-enriched phase. As shown in Fig. 5A, the unambiguous phase assignment was carried out by determining the partitioning of GM1, a ganglioside frequently used to identify sphingolipid-enriched rafts (Harder et al., 1998). Upon incubation of GUVs with a fluorescent conjugate of cholera toxin B subunit (CTB), for which GM1 is the natural receptor, the complex GM1-CTB was detected only in areas from which DiI$\mathrm{C}_{18}$ was strongly excluded (SM-enriched). Inclusion of as little as $10 \mathrm{~mol} \%$ cholesterol in the SM/DOPC $(0.5 / 0.5)$ bilayer sufficed to induce lipid segregation, as evidenced by the preferential partitioning of DiI- $\mathrm{C}_{18}$ in one phase (red areas in Fig. 5A) by a factor of $\sim 50$. Alexa Fluorlabeled cholera toxin bound to areas in the GUVs from which DiI- $\mathrm{C}_{18}$ was excluded and formed fluorescent regions exactly complementary to the ones covered by DiI$\mathrm{C}_{18}$ (green areas in Fig. 5A). Phase separation was also visualized at higher amounts of cholesterol (SM/DOPC $0.5 / 0.5$ ), for 20 and $33 \mathrm{~mol} \%$ cholesterol. The domain morphology was the same as for $10 \mathrm{~mol} \%$ cholesterol, except that the total surface area of the SM-enriched phase increased with the amount of cholesterol. At 50 and $65 \mathrm{~mol} \%$ cholesterol, rafts were no longer observed within the optical resolution (Fig. 5B).

Cholesterol is proven here to be the determining factor in causing phase separation of sphingolipids and unsaturated phospholipids. Unilamellarity of the vesicles allowed us to look for phase interlayer coupling and it was found that, in all GUVs, the phase domains comprised both apposing membrane leaflets. The long 
fatty acid chains of SM in opposite leaflets are likely able to superimpose by interdigitation, demonstrating that the lipid component alone can create strong coupling between inner and outer leaflet.

Consistent with previous studies reporting phase separation in model membranes with similar lipid mixtures (Dietrich et al., 2001a,b; Keller et al., 2002), the round shape of the domains suggests the coexistence of fluid phases, as the circular borders of the domains minimize the line energy. Domains with analogous patterns have been observed in mixtures that replace SM with DPPC or DSPC (Scherfeld et al., 2003). An amount of $20 \mathrm{~mol} \%$ cholesterol needs to be added to DPPC/DOPC mixtures in order to generate a pattern of round-shaped domains, similar to that obtained for SM/ DOPC/cholesterol GUVs (see Fig. 5C). Domains vanish at cholesterol concentrations of $33 \mathrm{~mol} \%$ and higher. Replacing DPPC with DSPC, extensive lipid segregation takes place at $20 \mathrm{~mol} \%$ (Fig. 5D) cholesterol and persists at $33 \mathrm{~mol} \%$ cholesterol (Scherfeld et al., 2003). Evidently, the tendency to form extensive domains is weaker for DOPC/DPPC/cholesterol (only at $20 \%$ cholesterol) than for DOPC/DSPC/cholesterol (between 20 and $33 \%$ cholesterol). In both cases, this tendency is much weaker than for the DOPC/SM/cholesterol mixture (between 10 and 33\% cholesterol). This reflects weaker DPPC(DSPC)/cholesterol with respect to SM/ cholesterol interactions, as explained under Sphingomyelin compared to saturated phospholipids.

Sizes of artificial rafts are consistently on the micrometer scale $(1-50 \mu \mathrm{m})$, in striking contrast to the putative raft size in cells $(\sim 20-500 \mathrm{~nm})$, in which rafts are difficult to visualize. This often raises the question whether what we observe in GUVs actually corresponds to a good model for rafts in vivo. However, in analogy to what is observed for rafts in cells, depletion and reconstitution of cholesterol in artificial membranes composed of SM/DOPC/cholesterol (1/1/1) resulted in disappearance and reappearance of lipid domains (Dietrich et al., 2001a,b). Our FCS data (Kahya et al., 2003) clearly show that domains in GUVs composed of $\mathrm{SM} / \mathrm{DOPC} /$ cholesterol exist in the liquid-ordered phase (see the following section), thought to be the fluid state of rafts in living cells (Brown and London, 1998). This and other experimental evidence indicate that domains, as visualized in GUVs, represent an accurate model for rafts and allow for investigation of raft-like properties under controllable conditions.

\section{Cholesterol strongly modulates lipid mobility in domain- forming giant unilamellar vesicles}

Cholesterol is present at high concentrations in the plasma membrane and plays an important role in membrane phase behavior. Its ability to intercalate with sphingolipids and thereby strongly affect the lipid packing has been shown to lead to raft assembly. The structural changes induced by lipid-sterol interactions result in a different lipid organization in the membrane and also, importantly, in substantial changes in lipid mobility. As lipid rafts are thought to be dynamic assemblies in membranes, the assessment of lipid dynamic properties is an important step toward the understanding of how lipids modulate membrane fluidity and thereby possibly control the timing of cellular events, such as sorting or signaling. Despite the enormous amount of data available on lipid rafts and domain physicochemical properties in model membranes, very few studies have so far focused on the lipid dynamic properties of membranes that exhibit domain formation. In addition, most of these studies employ FRAP, which requires high amounts of the fluorescent probe, thereby possibly altering the lipid organization (Schwille et al., 1999; Yuan et al., 2002). In contrast, FCS provides quantitative information on the average number of particles in focus and their dynamic properties, e.g., diffusion coefficients, with single-molecule sensitivity. This is a great advantage in studies on domain formation in membranes, as (i) lipid analogs can be introduced in minimal amounts and (ii) at the single molecule level, clustering of the dye may be readily detected. Furthermore, FCS has been shown to be sensitive to deviations from single-phase behavior, e.g., caused by heterogeneities in the sample (Korlach et al., 1999). Importantly, previous studies on lipid dynamics mostly refer to model membranes such as supported bilayers and/or multibilayer vesicles, which show artifacts in the diffusion properties and/or report only on the properties of domains overlapping in the multilayer membrane. Truly unilamellar vesicles, such as those prepared by electroformation, provide single free-standing bilayers in the focal volume.

Recently, we have employed FCS to systematically analyze the effect of cholesterol on lipid mobility in raftexhibiting GUVs made from ternary mixtures of DOPC/ $\mathrm{SM} /$ cholesterol, by following the fluorescence signal from DiI-C 18 (Kahya et al., 2003). The results point toward the important role of cholesterol in tuning the lipid mobility in raft-containing membranes (see Fig. 6A). In the case of phase separation, the corresponding diffusion coefficients of DiI- $\mathrm{C}_{18}$ are reported for the bright phase (Fig. 6A, red), where DiI- $\mathrm{C}_{18}$ preferentially partitioned, and the dark phase (Fig. 6A, black). The bright phase is characterized by very high lipid mobility, close to that in GUVs made from pure DOPC, and can be, therefore, assigned as the liquid-disordered, DOPC-enriched phase. On the other hand, DiI-C 18 -depleted domains show very low lipid mobility and are thus assigned as the liquidordered, SM-enriched domain. Consistent with previous studies (Dietrich et al., 2001b), lipid diffusion in the liquid-disordered phase was found approximately two 
times faster than in cholesterol entirely devoid GUVs (SM/DOPC 0.5/0.5). Most importantly, in contrast to some previous FRAP measurements (Dietrich et al., 2001a), we observed a remarkable change in lipid diffusion upon increase of sterol amount in the raft phases (SM-enriched). Here, the mobility increased by a factor of $\sim 8$, as the cholesterol concentration increased from 10 up to $33 \%$. This implies that cholesterol not only acts as raft promoter in association with sphingomyelin, but is also able to modulate lipid dynamics in domains (Kahya et al., 2003).

Given the data reproducibility, the good statistical accuracy of the results, and the excellent properties of GUVs as model membranes, the diffusion coefficients, as measured by FCS in the context of a particular lipid mixture, uniquely characterize a certain lipid phase composition. On the basis of the FCS measurements combined with the data from confocal imaging, we constructed a rough lipid phase diagram for the ternary mixture DOPC/SM/cholesterol (Fig. 6B). With the help of such a phase diagram, one can compare membrane fluidity for different lipid ratios and extract information on the phase lipid composition.

\section{Sphingomyelin compared to saturated phospholipids: raft assembly and membrane fluidity}

In recent years, the effects of cholesterol on the packing of sphingo- and phospholipids have been the main goal of intensive research (see for review OhvoRekilä et al., 2002; McConnell and Vrljic, 2003). Since saturated phospholipids show properties similar to those of sphingolipids, the question whether saturated phospholipids could intercalate with cholesterol and form rafts in the plasma membrane is a very debated one (Ohvo-Rekilä et al., 2002). Besides, if this is the case, then what is the peculiar role of sphingolipid in regulating raft formation? The interfacial region of saturated phospholipids significantly differs from that of SM, mainly because the amide group present in SM can act as hydrogen-bond acceptor, a feature absent from phospholipids (Brown and London, 1998; Ramstedt and Slotte, 1999, 2002). In the past, conventional techniques, e.g., detergent insolubility, have been often used to reveal rafts in vitro and in vivo. However, they did not show significant differences between sphingomyelin-enriched and saturated phospholipid (i.e., DPPC)-enriched phases (Ahmed et al., 1997; Nyholm and Slotte, 2001). Furthermore, few data have been reported on lipid dynamics and membrane viscosity in ternary mixtures of phospholipids and cholesterol. In our laboratory, domain formation was investigated in GUVs obtained from DOPC/DPPC/cholesterol and DOPC/DSPC/cholesterol mixtures at various compositions, in order to compare the effect of sphingomyelin on lipid dynamics in raft-like domains with that of saturated phospholipids, i.e., DPPC and DSPC. Membrane fluidity in GUVs made of DOPC/DPPC/cholesterol and DOPC/DSPC/ cholesterol at various compositions has been recently characterized by FCS following the fluorescence from DiI-C ${ }_{18}$ (Scherfeld et al., 2003). Lipid mobility for the DOPC/DPPC/cholesterol system varies significantly as a function of the sterol content (Fig. 7A). In the presence of lipid segregation ( $20 \%$ cholesterol), liquid-ordered, DPPC/cholesterol-enriched domains, from which DiI$\mathrm{C}_{18}$ is largely excluded, are characterized by very slow dynamics, whereas bright regions present a lipid mobility analogous to that of pure DOPC. In the case of domain assembly for DOPC/DSPC/cholesterol, the partitioning behavior of DiI- $\mathrm{C}_{18}$ is opposite that of the DOPC/DPPC/cholesterol system, as it favors the DSPCenriched region (very slow lipid dynamics, see Fig. 7B, red) over the DOPC-enriched region (Fig. 7B, black).

Addition of cholesterol to DOPC/DPPC or DOPC/ DSPC exerts the most significant effect on the lipid dynamics of DSPC- and DPPC-enriched domains rather than on that of the DOPC-enriched phase. This indicates a preference for saturated phospholipids over unsaturated ones. Extensive studies on phospholipid/ cholesterol interactions, in which cholesterol is shown to interact more strongly with saturated than with unsaturated PCs, support our conclusions (Brzustowicz et al., 2002; Ohvo-Rekilä et al., 2002). FCS allows for a quantitative comparison of lipid lateral diffusion between DPPC-enriched and DSPC-enriched domains, showing that cholesterol "fluidizes" the lipid bilayer more in the case of DSPC than for DPPC. The same behavior of cholesterol is also observed in the SM-enriched raft-like domains in SM/DOPC/cholesterol GUVs, but the effect on the lateral diffusion of SM-enriched regions is much stronger than for the saturated PCs (Kahya et al., 2003).

Overall, it is evident from confocal imaging and FCS measurements that, by replacing sphingomyelin with saturated phospholipids (with matched chain length) in ternary mixtures with DOPC and cholesterol, lipids can still assemble into domains but more cholesterol is required. Also, the molecular packing properties and the diffusion dynamics reflect weaker PC/cholesterol interactions compared to $\mathrm{SM} /$ cholesterol.

\section{Conclusions and outlook}

FCS has been shown to provide detailed information on the lipid dynamic properties of lipid mixtures. Lateral diffusion coefficients in membranes serve as a parameter to characterize molecular packing in membranes and ultimately lipid-lipid and/or lipid-sterol interactions. They also give insight into the lipid composition of distinct domains. In fact, even if phase separation is not evident on 
the confocal image because of the weak contrast created by the partitioning of the lipid probe, FCS might provide direct evidence for the existence of domain formation.

The ultimate goal is to study the lateral organization and association/dissociation equilibrium of (raft-associated) membrane proteins and their interplay with the lipid components. However, the GUVs' fragility and preparation procedures have until recently precluded a correct and controlled insertion of proteins in such bilayers. A reconstitution protocol was recently developed, based upon membrane fusion between GUV and protein-reconstituted submicrometer vesicles, showing that membrane proteins can be effectively incorporated into GUVs (Kahya et al., 2001, 2002). This and other techniques, currently under development in our laboratory, should prove to be extremely useful in addressing fundamental questions regarding lipid-protein interactions, protein function, and dynamics in a controlled, though close-to-native, membrane matrix.

\section{Acknowledgments}

We thank Professor Dick Hoekstra for useful discussions and the Volkswagen Foundation for financial support of the project. Additionally, N.K. enjoyed a short-term EMBO fellowship in the early phase of the collaboration (ASTF66-2002).

\section{References}

Ahmed, S.N., Brown, D.E., London, E., 1997. On the origin of sphingolipid/cholesterol-rich detergent-insoluble cell membranes: physiological concentrations of cholesterol and sphingolipid induce formation of a detergent-insoluble, liquid-ordered lipid phase in model membranes. Biochemistry 36, 10944-10953.

Almeida, P.F.F., Vaz, W.L.C., Thompson, T.E., 1993. Percolation and diffusion in three-component lipid bilayers: effect of cholesterol on an equimolar mixture of two phosphatidylcholines. Biophys. J. 64, $399-412$.

Angelova, M.I., Dimitrov, D.S., 1986. Liposome electroformation. Faraday Discuss. Chem. Soc. 81, 303-308.

Angelova, M.I., Soléau, S., Méléard, P., Faucon, J.F., Bothorel, P., 1992. Preparation of giant vesicles by external AC electric fields: kinetics and applications. Prog. Colloid Polym. Sci. 89, 127-131.

Axelrod, D., Koppel, D.E., Schlessinger, J., Elson, E., Webb, W.W., 1976. Mobility measurements by analysis of fluorescence photobleaching recovery kinetics. Biophys. J. 16, 1055-1069.

Bacia, K., Majoul, I.V., Schwille, P., 2002. Probing the endocytic pathway in live cells using cross-correlation analysis. Biophys. J. 83, 1184-1193.

Bagatolli, L.A., Gratton, E., 1999. Two-photon fluorescence microscopy observation of shape changes at the phase transition in phospholipid giant unilamellar vesicles. Biophys. J. 77, 2090-2101.

Bagatolli, L.A., Gratton, E., 2000. A correlation between lipid domain shape and binary phospholipid mixture composition in free standing bilayers: a two-photon fluorescence microscopy study. Biophys. J. 79, 434-447.

Brown, D.A., 2001. Seeing is believing: visualization of rafts in model membranes. Proc. Natl. Acad. Sci. USA 98, 10517-10518.
Brown, D.A., London, E., 1998. Structure and origin of lipid domains in biological membranes. J. Membr. Biol. 164, 103-114.

Brown, D.A., London, E., 2000. Structure and function of sphingolipid- and cholesterol-rich membrane rafts. J. Biol. Chem. 275, 17221-17224.

Brown, D.A., Rose, J.K., 1992. Sorting of GPI-anchored proteins to glycolipid-enriched membrane subdomains during transport to the apical cell surface. Cell 68, 533-544.

Brzustowicz, M.R., Cherezov, V., Zerouga, M., Caffrey, M., Stillwell, W., Wassall, S.R., 2002. Controlling membrane cholesterol content. a role for polyunsaturated (docosahexaenoate) phospholipids. Biochemistry 41, 12509-12519.

Demel, R.A., de Kruijff, B., 1976. The function of sterols in membranes. Biochim. Biophys. Acta 457, 109-132.

Dietrich, C., Bagatolli, L.A., Volovyk, Z.N., Thompson, N.L., Levi, M., Jacobson, K., Gratton, E., 2001a. Lipid rafts reconstituted in model membranes. Biophys. J. 80, 1417-1428.

Dietrich, C., Volovyk, Z.N., Levi, M., Thompson, N.L., Jacobson, K., 2001b. Partitioning of Thy-1, GM1, and cross-linked phospholipid analogs into lipid rafts reconstituted in supported model membrane monolayers. Proc. Natl. Acad. Sci. USA 98, 10642-10647.

Edidin, M., 2003. Timeline: lipids on the frontier: a century of cellmembrane bilayers. Nat. Rev. Mol. Cell Biol. 4, 415-418.

Eigen, M., Rigler, R., 1994. Sorting single molecules: application to diagnostics and evolutionary biotechnology. Proc. Natl. Acad. Sci. USA 91, 5740-5747.

Fahey, P.F., Koppel, D.E., Barak, L.S., Wolf, D.E., Elson, E.L., Webb, W.W., 1977. Lateral diffusion in planar lipid bilayers. Science 195, 305-306.

Feigenson, G.W., Buboltz, J.T., 2001. Ternary phase diagram of dipalmitoyl-PC/dilauroyl-PC/cholesterol: nanoscopic domain formation driven by cholesterol. Biophys. J. 80, 2775-2788.

Fridriksson, E.K., 1999. Quantitative analysis of membrane domains in functionally important membrane domains from RBL-2H3 mast cells using tandem high-resolution mass spectrometry. Biochemistry $38,8056-8063$.

Fujiwara, T., Ritchie, K., Murakoshi, H., Jacobson, K., Kusumi, A., 2002. Phospholipids undergo hop diffusion in compartmentalized cell membrane. J. Cell Biol. 157, 1071-1081.

Harder, T., Scheiffele, P., Verkade, P., Simons, K., 1998. Lipid domain structure of the plasma membrane revealed by patching of membrane components. J. Cell Biol. 141, 929-942.

Heerklotz, H., 2002. Triton promotes domain formation in lipid rafts mixtures. Biophys. J. 83, 2693-2701.

Holopainen, J.M., Angelova, M.I., Kinnunen, P.K.J., 2000. Vectorial budding of vesicles by asymmentrical enzymatic formation of ceramide in giant liposomes. Biophys. J. 78, 830-838.

Huang, J., Buboltz, J.T., Feigenson, G.W., 1999. Maximum solubility of cholesterol in phosphatidylcholine and phosphatidylethanolamine bilayers. Biochim. Biophys. Acta 1417, 89-100.

Ipsen, J.H., Karlstrom, G., Mouritsen, O.G., Wennerstrom, H., Zuckermann, M.J., 1987. Phase equilibria in the phosphatidylcholine-cholesterol system. Biochim. Biophys. Acta 905, 162172.

Jain, M.K., 1972. The Bimolecular Lipid Membrane: A System. Litton Educational Publ., New York. pp. 52-144.

Kahya, N., Pécheur, E.-I., de Boeij, W.P., Wiersma, D.A., Hoekstra, D., 2001. Reconstitution of membrane proteins into giant unilamellar vesicles via peptide-induced fusion. Biophys. J. 81, 1464 1474.

Kahya, N., Scherfeld, D., Bacia, K., Poolman, B., Schwille, P., 2003. Probing lipid mobility of raft-exhibiting model membranes by fluorescence correlation spectroscopy. J. Biol. Chem. (in press).

Kahya, N., Wiersma, D.A., Poolman, B., Hoekstra, D., 2002. Spatial organization of bacteriorhodopsin in model membranes: lightinduced mobility changes. J. Biol. Chem. 277, 39304-39311. 
Kenworthy, A.K., Petranova, N., Edidin, M., 2000. Mol. Biol. Cell 11, $1645-1655$.

Koppel, D.E., 1974. Statistical accuracy in fluorescence correlation spectroscopy. Phys. Rev. A 10, 1938-1945.

Korlach, J., Schwille, P., Webb, W.W., Feigenson, G.W., 1999. Characterization of lipid bilayer phases by confocal microscopy and fluorescence correlation spectroscopy. Proc. Natl. Acad. Sci. USA 96, 8461-8466.

Lipp, M.M., Lee, K.Y.C., Zasadzinski, J.A., Waring, A.J., 1996. Phase and morphology changes in lipid monolayers induced by SP-B protein and its amino-terminal peptide. Science 273, 1196-1199.

Magde, D., Elson, E., Webb, W.W., 1972. Thermodynamic fluctuations in a reacting system: measurement by fluorescence correlation spectroscopy. Phys. Rev. Lett. 29, 705-708.

McConnell, H.M., Radhakrishnan, A., 2003. Condensed complexes of cholesterol and phospholipids. Biochim. Biophys. Acta 1610, 159173.

McConnell, H.M., Vrljic, M., 2003. Liquid-liquid immiscibility in membranes. Annu. Rev. Biophys. Biomol. Struct. 32, 469-492.

Menger, F.M., Keiper, J.S., 1998. Chemistry and physics of giant vesicles as biomembrane models. Curr. Opin. Chem. Biol. 2, 726-732.

Milon, S., Hovius, R., Vogel, H., Wohland, T., 2003. Factors influencing fluorescence correlation spectroscopy measurements on membranes: simulations and experiments. Chem. Phys. 288, 171-186.

Needham, D., Stoicheva, N., Zhelev, D.V., 1997. Exchange of monooleoylphosphatidylcholine as monomer and micelle with membranes containing poly(ethylene glycol)-lipid. Biophys. J. 73, 2615-2629.

Ohvo-Rekilä, H., Ramstedt, B., Leppimäki, P., Slotte, J.P., 2002. Cholesterol interactions with phospholipids in membranes. Prog. Lipid Res. 41, 66-97.

Ramstedt, B., Slotte, J.P., 1999. Interaction of cholesterol with sphingomyelins and acyl-chain-matched phosphatidylcholines: a comparative study of the effect of the chain length. Biophys. J. 76, 908-915.

Ramstedt, B., Slotte, J.P., 2002. Membrane properties of sphingomyelins. FEBS Lett. 531, 33-37.

Rarbach, M., Kettling, U., Koltermann, A., Eigen, M., 2001. Dualcolor fluorescence cross-correlation spectroscopy for monitoring the kinetics of enzyme-catalyzed reactions. Methods 24, 104-116.

Sackmann, E., 1996. Supported membranes: scientific and practical applications. Science 271, 5245-5248.

Sankaram, M.B., Thompson, T.E., 1991. Cholesterol-induced fluidphase immiscibility in membranes. Proc. Natl. Acad. Sci. USA 88 , 8686-8690.

Saxton, M.J., Jacobson, K., 1997. Single particle tracking: applications to membrane dynamics. Annu. Rev. Biophys. Biomol. Struct. 26, 373-399.

Scherfeld, D., Kahya, N., Bacia, K., Schwille, P., 2003. Lipid dynamics and domain formation in model membranes composed of ternary mixtures of saturated and unsaturated phosphatidylcholines and cholesterol. Biophys. J. (in press).
Schütz, G.J., Kada, G., Pastushenko, V.P., Schindler, H., 2000. Properties of lipid microdomains in a muscle cell membrane visualized by single molecule microscopy. EMBO J. 19, 892-901.

Schwille, P., 2001. Fluorescence correlation spectroscopy and its potential for intracellular applications. Cell Biochem. Biophys. 34, 383-408

Schwille, P., Korlach, J., Webb, W.W., 1999. Fluorescence correlation spectroscopy with single molecule sensitivity on cell and model membranes. Cytometry 36, 176-182.

Schwille, P., Meyer-Almes, F.-J., Rigler, R., 1997. Dual-color fluorescence cross-correlation spectroscopy for multicomponent diffusional analysis in solution. Biophys. J. 72, 1878-1886.

Seifert, U., 1997. Configurations of fluid membranes and vesicles. Adv. Phys. 46, 13-137.

Silvius, J.R., 2003. Role of cholesterol in lipid raft formation: lessons from lipid model systems. Biochim. Biophys. Acta 1610, 174-183.

Silvius, J.R., del Giudice, D., Lafleur, M., 1996. Cholesterol at different bilayer concentrations can promote or antagonize lateral segregation of phospholipids of differing acyl chain length. Biochemistry 35, 15198-15208.

Simons, K., Ikonen, E., 1997. Functional rafts in cell membranes. Nature 387, 569-572.

Simons, K., Toomre, D., 2000. Lipid rafts and signal transduction. Nat. Rev. Mol. Cell Biol. 1, 31-39.

Simons, K., van Meer, G., 1988. Lipid sorting in epithelial cells. Biochemistry 27, 6197-6202.

Spink, C.H., Yeager, M.D., Feigenson, G.W., 1990. Partitioning behavior of indocarbocyanine probes between coexisting gel and fluid phases in model membranes. Biochim. Biophys. Acta 1023, $25-33$.

Sprong, H., van der Sluijs, P., van Meer, G., 2001. How proteins move lipids and lipids move proteins. Nat. Rev. Mol. Cell Biol. 2, 504 513

Ueda, T., Lee, S.J., Nakatani, Y., Ourisson, G., Sunamoto, J., 1998. Coating of POPC liposomes with hydrophobized polysaccharide. Chem. Lett. 27, 417-418.

von Tscharner, V., McConnell, H.M., 1981. An alternative view of phospholipid phase behavior at the air-water interface: microscope and film balance studies. Biophys. J. 36, 409-419.

Vist, M.R., Davis, J.H., 1990. Phase equilibria of cholesterol/ dipalmitoylphosphatidylcholine mixtures: ${ }^{2} \mathrm{H}$ nuclear magnetic resonance and differential scanning calorimetry. Biochemistry 29, $451-464$

Wick, R., Angelova, M.I., Walde, P., Luisi, P.L., 1996. Microinjection into giant vesicles and light microscopy investigation of enzymemediated vesicle transformations. Chem. Biol. 3, 105-111.

Yeagle, P.L., Hutton, W.C., Huang, C., Martin, R.B., 1977. Phospholipid head-group conformations; intermolecular interactions and cholesterol effects. Biochemistry 16, 4344-4349.

Yuan, C., Furlong, J., Burgos, P., Johnston, L.J., 2002. The size of lipid rafts: an atomic force microscopy study of ganglioside GM1 domains in sphingomyelin/DOPC/cholesterol membranes. Biophys. J. $82,2526-2535$. 